\title{
Cosmetic textiles with biological benefits: Gelatin microcapsules containing Vitamin C
}

\author{
SHUK YAN CHENG ${ }^{1}$, MARCUS CHUN WAH YUEN ${ }^{1}$, CHI WAI KAN ${ }^{1}$, \\ KEVIN KA LEUNG CHEUK ${ }^{1}$, CHUNG HIN CHUI ${ }^{1}$ and KIM HUNG LAM ${ }^{2}$ \\ ${ }^{1}$ Institute of Textiles and Clothing, ${ }^{2}$ Department of Applied Biology and Chemical Technology, \\ The Hong Kong Polytechnic University, Hong Kong, P.R. China
}

Received May 21, 2009; Accepted July 2, 2009

DOI: $10.3892 /$ ijmm_00000247

\begin{abstract}
In recent years, textile materials with special applications in the cosmetic field have been developed. A new sector of cosmetic textiles is opened up and several cosmetic textile products are currently available in the market. Microencapsulation technology is an effective technique to control the release properties of active ingredients that prolong the functionality of cosmetic textiles. This study discusses the development of cosmetic textiles and addresses microencapsulation technology with respect to its historical background, significant advantages, microencapsulation methods and recent applications in the textile industry. Gelatin microcapsules containing vitamin $\mathrm{C}$ were prepared using emulsion hardening technique. Both the optical microscopy and scanning electron microscopy demonstrated that the newly developed microcapsules were in the form of core-shell spheres with relatively smooth surface. The particle size of microcapsules ranged from 5.0 to $44.1 \mu \mathrm{m}$ with the average particle size being $24.6 \mu \mathrm{m}$. The gelatin microcapsules were proved to be non-cytotoxic based on the research findings of the toxicity studies conducted on human liver and breast cell lines as well as primary bone marrow culture obtained from patient with non-malignant haematological disorder. The gelatin microcapsules were successfully grafted into textile materials for the development of cosmetic textiles.
\end{abstract}

\section{Contents}

1. Introduction

2. Cosmetic textiles

3. Microencapsulation

Correspondence to: Professor Marcus Chun Wah Yuen, Institute of Textiles and Clothing, The Hong Kong Polytechnic University, Yuk Choi Road, Hung Hom, Kowloon, Hong Kong, P.R. China E-mail: tcyuencw@inet.polyu.edu.hk

Key words: cosmetic textiles, microcapsules, biological benefits, in vitro toxicity test
4. Synthesis of gelatin/vitamin C microcapsules and its application on textile materials

5. Conclusion

\section{Introduction}

People have always tried to improve their quality of life with positive developments in hygiene and nutrition as a result of rapid development in medical knowledge. The developments nowadays are focused not only on the solutions to medical problems, but also on the possible ways to improve the comfort of application. Textile systems may be useful in this aspect (1).

The typical benefit obtained from the use of textile materials is protection. However, with the growing demand of enhancing beauty with health, customers request that the apparels and home textiles not only have the original basic characteristics such as maintaining warmth and providing comfort, but also carry extra functions including environmental protection, anti-pollution and the most concerned function like health and beauty care in an attempt to provide a more natural and healthier life $(1,2)$.

The breathing capability makes textile materials become a useful basis for ex vivo applications (1). A brainstorming idea has been developed to make textile materials useful in the cosmetic field. As people are getting used to wearing textile materials in their daily life, it is also logical to use textile as the possible basis for cosmetic substance delivery system (1). A new terminology, so called 'cosmetic textiles' has now been introduced to the textile industry and it is very optimistic that these products will open up new target groups and sustainable markets $(1,3,4)$.

\section{Cosmetic textiles}

On contact with the skin and human body, cosmetic textiles are designed to transfer an active substance for cosmetic purposes. One particular example is the transfer of skinmoisturising substances from the cosmetic textiles to human body. The principle is achieved by simply imparting the cosmetic and pharmaceutical ingredients into the fabric of garment so that with the natural movement of body, the skin is slowly freshened and revitalised. In order to achieve the functional effects, microencapsulation technology appears to 


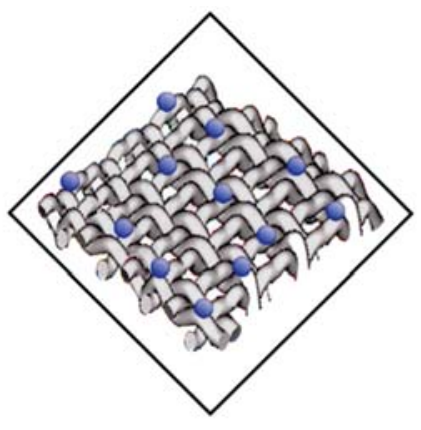

Microcapsules on fabric

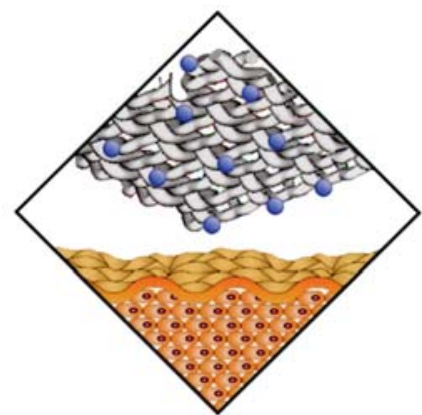

Contacting the skin

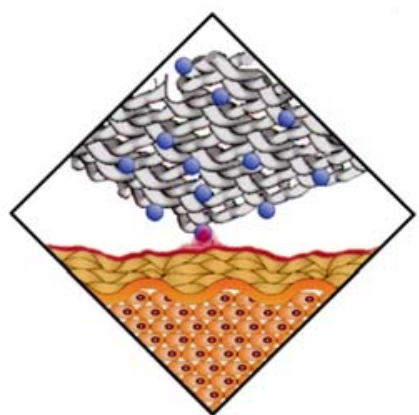

Ingredients are released

Figure 1. Working mechanism of cosmetic textiles.

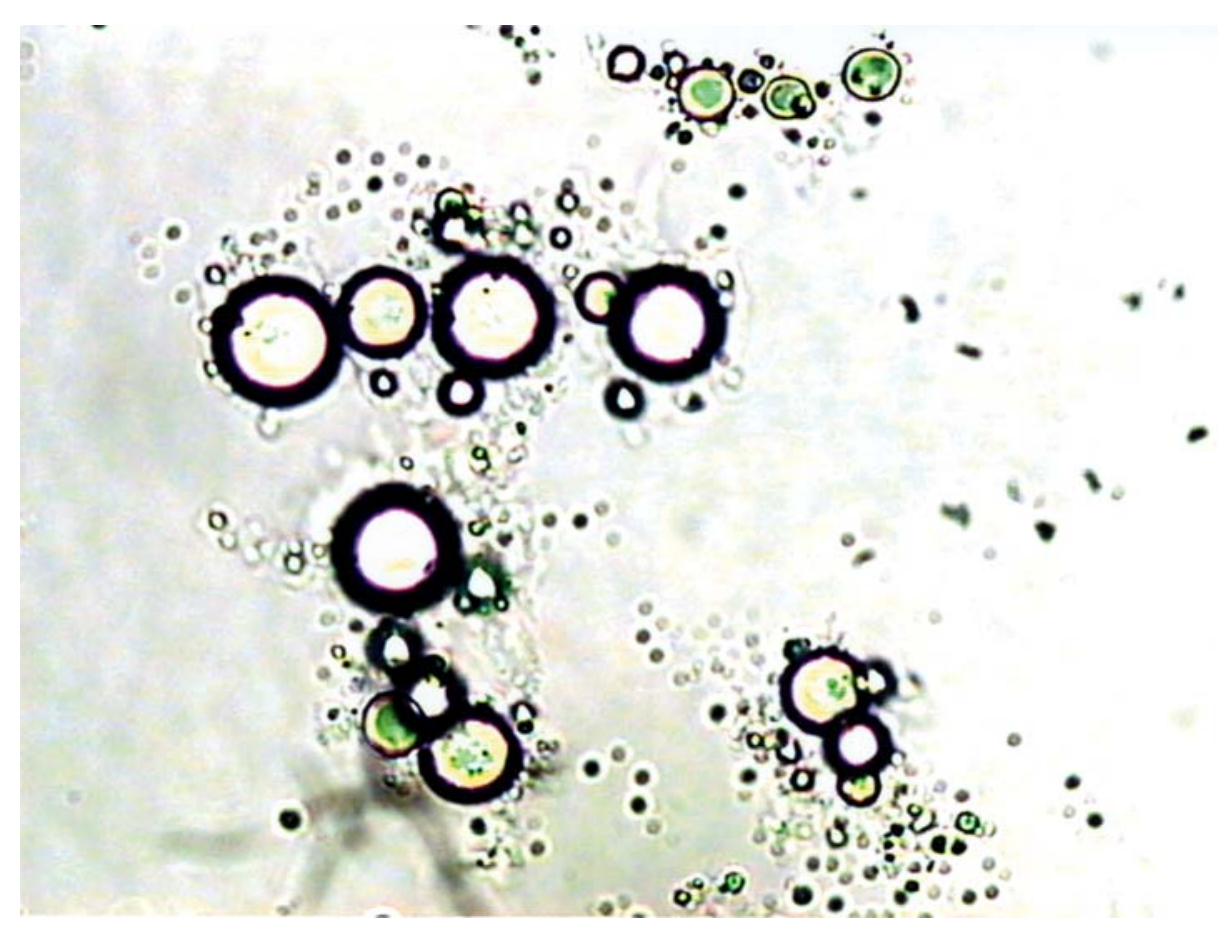

Figure 2. Optical image of chitosan microcapsules containg jojoba oil.

be an alternative way to provide satisfactory performance with increased durability. Fig. 1 illustrates the working mechanism of cosmetic textiles when contacting the skin.

The controlled release properties of the active ingredient with respect to the cosmetic textiles is an important aspect to be studied. Under a slow-release delivery mechanism, the cosmetic substance can be released to human skin in a controlled way to avoid overdose $(1,5)$.

The biocompatibility of the cosmetic substance delivery system is another concern in the development of cosmetic textile. Biocompatibility can be defined as the ability of a material to perform with an appropriate host response in a specific application. The cosmetic substance delivery system of cosmetic textiles should be non-toxic and non-carcinogenic with no interest in the related issues of mutagenicity and teratogenicity. As an ex vivo application, the cosmetic textiles should not cause any irritation of skin at the same time of release the active ingredients (1).
In view of the increasing demand in the relevant fields, researchers and textile manufacturers have substantially invested in the cosmetic textiles for research and product development. In general, the events are focused on a) opportunity and limit for cosmetic and wellness applications of textiles, b) possible ways of incorporating active substances in a functional manner with increased durability, c) systematic characterisation model for assessing the cosmetic textiles for safety and d) biological safety and biological benefit to human skin.

\section{Microencapsulation}

Microencapsulation technology. Microencapsulation technology is now rapidly developing in the field of chemical finishing because of its versatility and flexibility. Microencapsulation is actually a micropackaging technique involving the production of microcapsules which act as 
Table I. Typical wall materials used for microencapsulation.

Types of wall material

Natural

Semi-synthetic

\section{Examples}

Gelatin, agar, gum, sodium alginate, calcium alginate, dextran, fat and fatty acid starch, chitosan, casemate, stearm, sucrose, and wax

Cellulose acetate, cellulose nitrate, ethylcellulose and hydroxypropylcellulose, methylcellulose, sodium carboxymethylcellulose, hydrogenated tallow, myristyl alcohol, glycerylomon-or dipalmitate, hydrogenated easter oil, glycerylmono-,di, or tristearate, and 12-hydroxystearyl alchol

Synthetic barrier walls of solids or liquids. The microcapsules are produced by depositing a thin polymer coating on small solid particles or liquid droplets, or on dispersions of solid in liquids. The core contents are released under the controlled conditions to suit a specific purpose. Particles in micro-size prepared by the microencapsulation technique are called microcapsules or microparticles (6-8). Fig. 2 and 3 show the optical image and scanning electron microscope (SEM) image of chitosan microcapsules containing essential oil.

The release mechanism of the core contents vary depending on the selection of wall materials and more importantly, its specific end uses. The core content may be released by friction, pressure, change of temperature, diffusion through the polymer wall, dissolution of the polymer wall coating or by biodegradation (9-11).

Historical background of microencapsulation. The earliest conception of microencapsulation, which carries or holds a core material trapped within a shell material, possibly dates back to 1930 s by using spray-drying technique (8). Until 1950 s, the first significant application of encapsulation technology was developed by Barrett Green of National Cash Register Company to provide carbonless copy paper using complex coacervation technique. This was employed in a novel printing system which incorporated a colourless dye within the oil phases and coated a second review article sheet with acidic clay $(8,12)$. Since then, the US-based Eurand America company had acquired the rights to subsequently develop and market microencapsulation technology for all new applications (13).

Microencapsulation techniques developed by a number of companies were noted henceforth. This versatile micropackaging technique has been applied in a wide range of fields including the pharmaceutical, bulk chemical, agricultural, food processing, cosmetic and toiletry industries $(6,13)$. The textile industry has reacted slowly to the possibilities of microencapsulation. It is not until 1990s that a few commercial applications were appearing at the research and development stages (6).

In the 21 st century, more commercial applications of microencapsulation in the textile industry can be found, particularly in Western Europe, Japan and North America. The technique is being used to develop textiles with new properties and added value; including climate-control materials, fragrances released fabrics, cosmetic, therapeutic

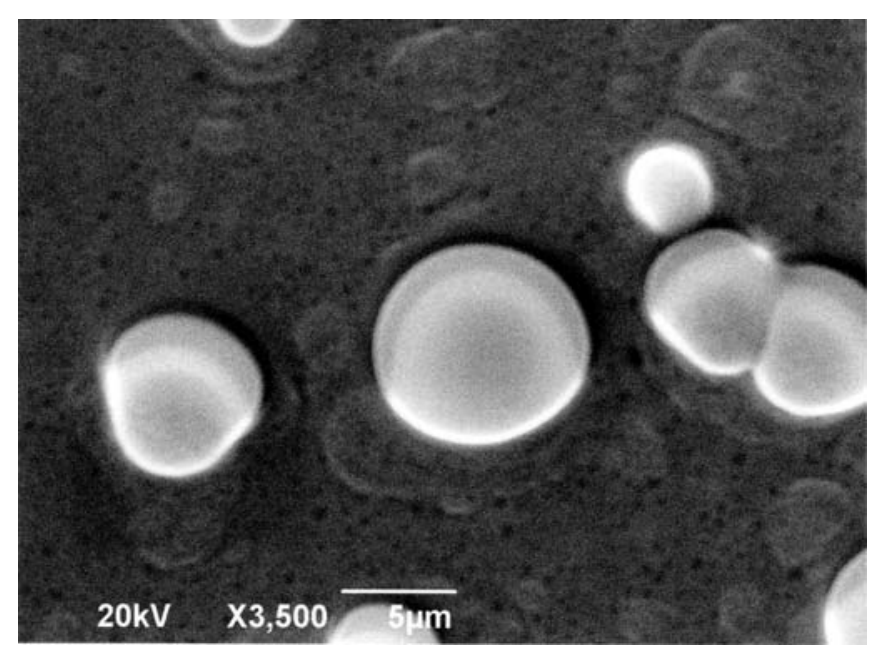

Figure 3. SEM image of chitosan microcapsules containing jojoba oil

and medical textiles. Textiles and garment manufactures are very optimistic that this novel technology will open up exciting market opportunities and a world of possibilities for consumers $(9,14-15)$.

Structure of microcapsules. Microcapsules are small liquid, solid or aerosol particles surrounded by another substance in order to protect, separate the materials and control the release of core materials. They may be considered as a way of storing materials at the microscopic level (16). Fig. 4 shows the general structure of microcapsule which generally consists of two major components:

i) Active ingredient. Active ingredient is the substance that may be in the form of liquid or solid. It is also referred to the core contents, internal phases, active, encapsulate, payload or fill (9).

ii) Wall shell. A polymer coating that surrounds the active ingredients which may also be called the wall, shell, external phase, membrane or matrix. It may be natural polymer, semisynthetic polymer and synthetic polymer (9).

Microcapsules can be made into various forms as shown in Fig. 5, but they are usually in spherical form. Microcapsules of irregular forms are known as a) spherical form with single nucleus, b) special form with multi nucleus, c) indeterminate form and d) indeterminate form with multi nucleus (16). 


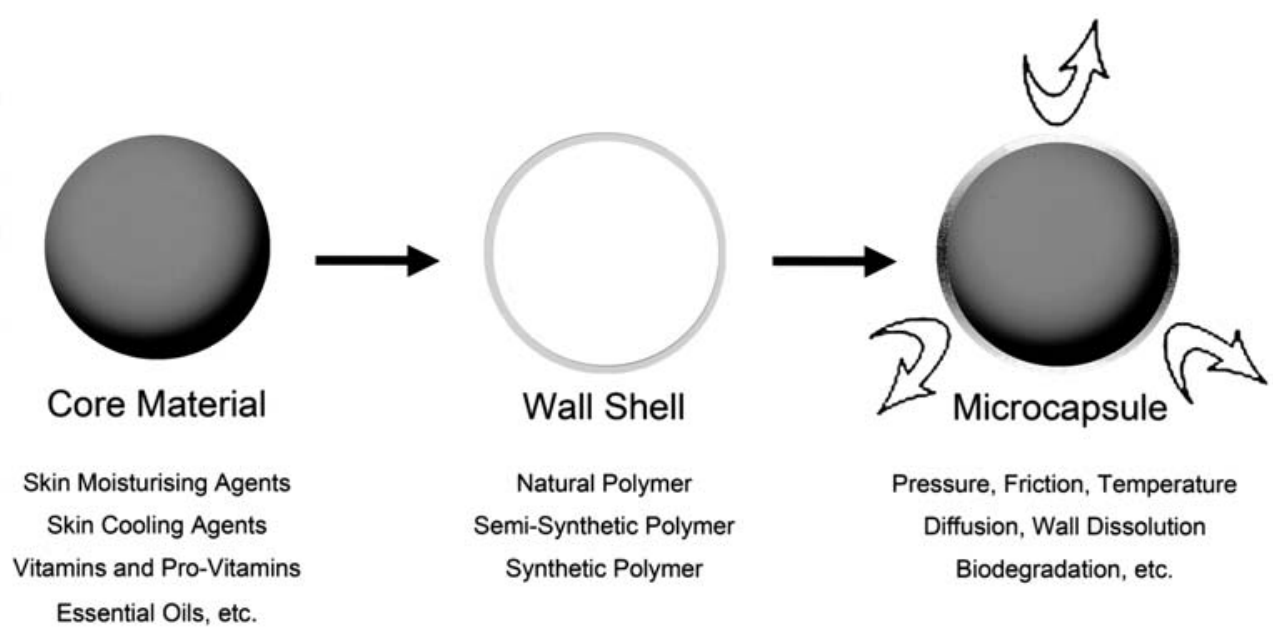

Figure 4. Structure of microcapsule.

Physical appearance of the microcapsule is generally governed by the physical nature of the core material. If solid or crystalline materials are encapsulated, the resulting microcapsule may have an irregular shape which tends to follow the original shape of the core particle. If a liquid is used for the core material, simple spherical microcapsules containing a single liquid droplet may be found (9).

Microcapsules can be produced with non-definite sizes and their typical diameters are in the range of 1-1,000 microns. Theoretically, microcapsules can be produced with diameters between 0.01 and 10,000 microns (9). The thickness of the microcapsule wall is generally in the range of $0.5-150$ microns. In most cases, the core content of the microcapsule will represent between 20 and $95 \%$ of the total mass (9).

The choice of wall materials depends upon a number of factors including expected product objectives, requirement, nature of the core material, the process of encapsulation and economics (17). The wall material may be natural, semisynthetic or synthetic. Table I illustrates the typical wall materials used for microencapsulation (12).

Microencapsulation methods. Many different manufacturing approaches have been adopted for microencapsulation. The most commonly used microencapsulation processes include a) complex coacervation, b) polymer-polymer incompatibility, c) interfacial polymerisation and in situ polymerisation, d) spray drying, e) centrifugal extrusion, f) air suspension coating, g) pan coating and $\mathrm{h}$ ) emulsion hardening process $(8,12,18,19)$.

Advantages of microencapsulation. One major advantage of using microencapsulation technology is its ability to protect active ingredients from hazardous environment, i.e. oxidisation, heat, acidity, alkalinity, moisture or evaporation. It also protects ingredients from interacting with other compounds in the system, which may result in degradation or polymerisation. At the same time, the use of microencapsulation technology also protects the microcapsule end users from exposure to hazardous substances such as pesticides. Another important advantage of this versatile technology is its controlled release properties that seem to be the best choice for increasing the efficiency and minimisation of environmental damage. Other advantages of microencapsulation technology include a) its ability to render liquids into powder form in order to prevent clumping and improve mixing, b) disguising the unpleasant flavours or odours such as pharmaceuticals as well as c) improving the handling of an ingredient before processing $(6,9,18-20)$.

Microencapsulation applications in cosmetic textiles. In cosmetic textiles, the major interest in microencapsulation is currently in the application of vitamins, essential oils, skin moisturising agents, skin cooling agents and anti-ageing agents etc. A few studies regarding the techniques of producing microcapsules containing cosmetic substances have been found in literature (21-28) (Table II).

Yamato et al prepared microcapsules comprising the active substances used to improve the physiological conditions of human skin. The microcapsule will not be broken during production, but is gradually released when the textiles structure is subjected to light pressure created by the movement of human body (21).

Bishop et al introduced the use of waste yeast cells in microencapsulation process. After encapsulating the core material, the yeast cells were attached to both cotton and wool fibre by using crosslinking agents and binders. The processes of filling the yeast cells were very simple and the use of yeast cells as wall material generally provided several advantages such as high loading, inthermoplastic, protection from light, oxygen and hazardous environment, and cost effectiveness (22).

Copete Vidal et al introduced the invention of chitosanbased microcapsules containing various active components and investigated the durability performance with the mixture of microcapsules and binding agent. With the effect of finishing using microcapsules and binder, the active ingredients were released and washed out less quickly, and a high degree of hydration was achieved as well (23).

Korean researchers prepared melamine resin microcapsules containing Migrin oil by in situ polymerisation method. The structure, mean particle size and size distribution, morphologies, thermal properties and released behaviour 
Table II. Typical core materials used for microencapsulation.

Types of core material

Solvents

Plasticiser

Acids and Bases

Catalysts

Colourants

Adhesives

Fragrances

Foods

Agricultural Chemicals

Pharmaceuticals

Recording Material

Rust Inhibitors

Others
Examples

Benzene, cyclohexane, chlorinated phenyls, paraffins, esters, ethers, alcohols and water

Phthalate, adipate and pjosphate-type, silicones and chlorinated hydrocarbons

Boric acid, caustic alkali and amines

Curing agents, oxidants, free radical initiators and reducing agents

Pigments and dyes

Polysulphides, cyanoacrylates and isocyanates

Menthol, essences and speciality compositions

Oils, fats, spices and flavours

Herbicides, insecticides and pesticides

Aspirin, vitamins and amino acids

Reprographic toners, coupling agents, developers, silver halides, fixing agents, photochromatic compound, liquid crystals

Zinc chromate and other compounds

Detergents, bleaches and fore retardant

A wide range of core materials including solvents, plasticisers, acids, bases and catalysts, etc can be encapsulated. Table II show some typical core materials to be encapsulated (12).

were characterised and discussed (24). They also prepared poly (L-lactide) microcapsules for fragrant fibre by interfacial precipitation method through solvent evaporation from water-in-oil-in-water emulsion. The microcapsules were then uniformly printed on the cotton fabrics and the resulted fabric could withstand 15 cycles of washing durability (25).

Jarfari et al have also tried to prepare microcapsules with polymethyl methacrylate (PMMA) as wall shell and Rose fragrance as core materials (26).

Many researchers have made efforts to improve the durability of microencapsulated functions. This is relatively the most difficult task in preparing the cosmetic textiles. Li et al investigated the effect of UV curing for the encapsulated aroma finish on cotton. The aroma function was prolonged to 50 wash cycles whereas the traditional curing method could withstand 25 wash cycles only (27). Chang integrated the processing procedure of fabric treatment with low temperature plasma, natural oil essence microencapsulation technique and fabric coating technique to improve the adhesion of microcapsules with fabrics (28).

\section{Synthesis of gelatin/vitamin $\mathrm{C}$ microcapsules and its application on textile materials}

To obtain a better understanding of the development of cosmetic textiles, gelatin microcapsules containing vitamin $\mathrm{C}$ were prepared in this study using the emulsion hardening technique. The gelatin microcapsules were subsequently

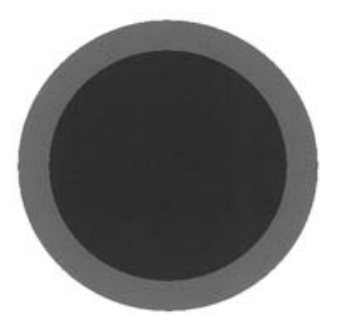

Spherical Form with Single Nucleus

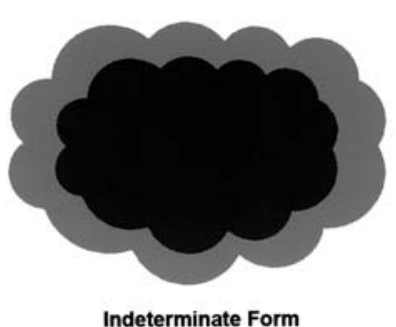

Figure 5. Forms of microcapsules.

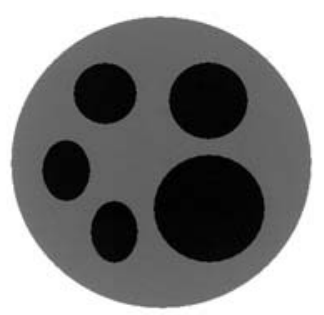

Spherical Form with Multi Nucleus

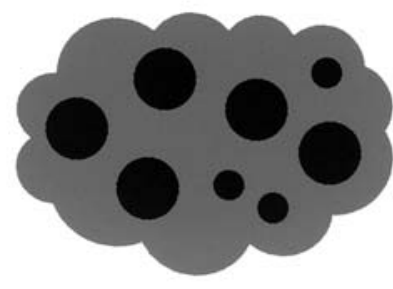

Indeterminate Form with Multi Nucleus grafted into fibrous materials for the production of cosmetic textiles.

Synthesis of gelatin microcapsules containing vitamin $C$. Gelatin (Ph Eur, Sigma Aldrich, St. Louis, MO) and vitamin C (Puriss. p.a, Sigma Aldrich) as shown in Fig. 6A and B, were dissolved in deionised water at the temperature of $40-60^{\circ} \mathrm{C}$ 
A

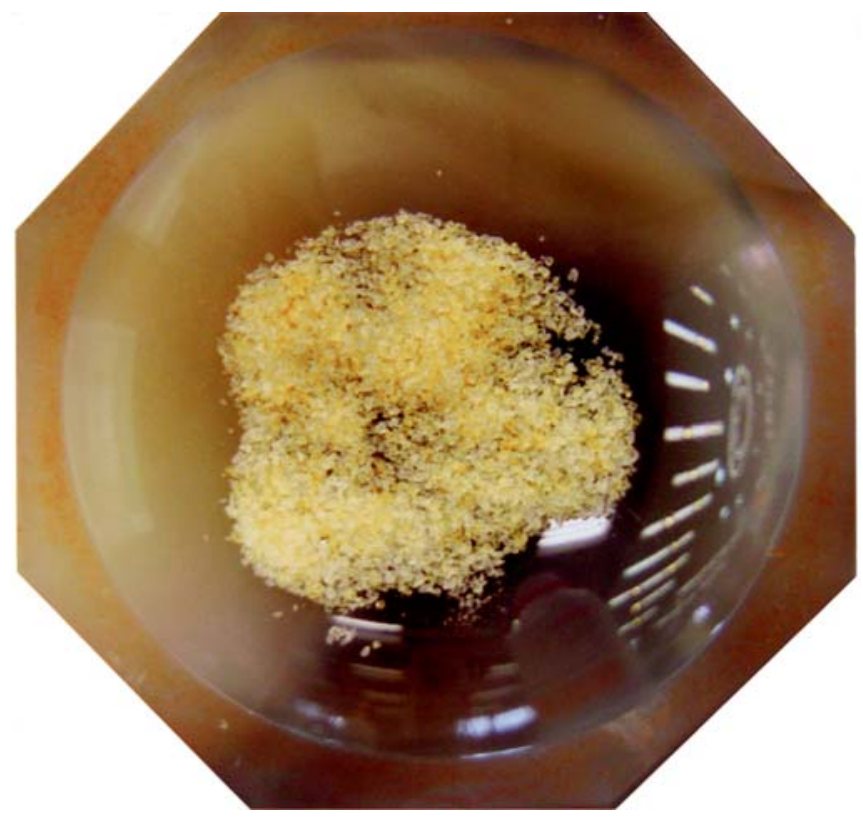

C

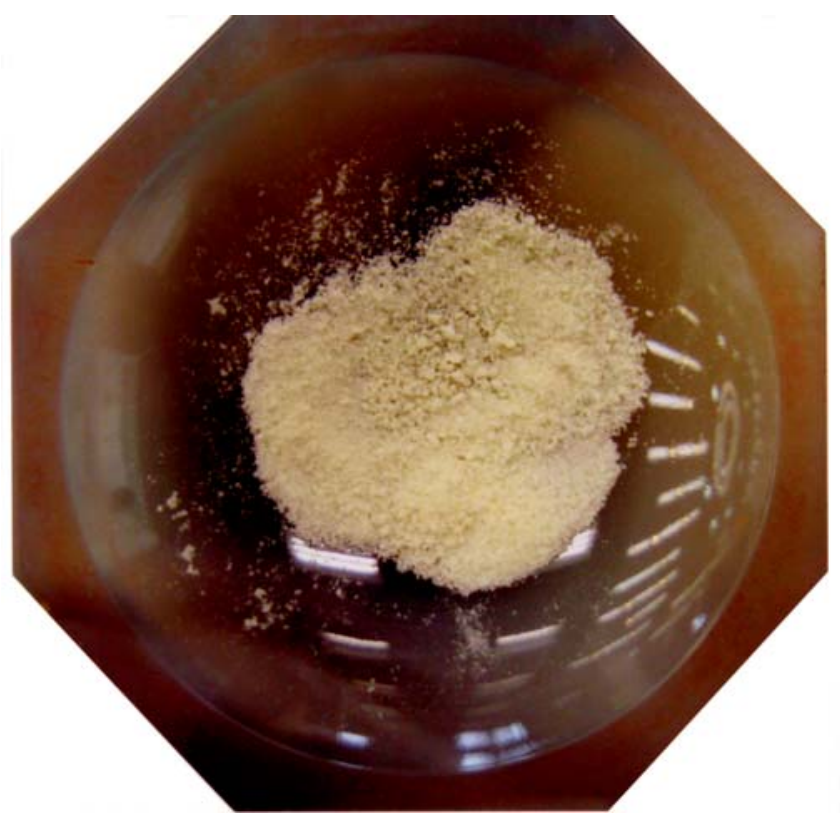

for $15 \mathrm{~min}$. The solution was then poured into $100 \%$ pure canola oil to form the water in oil emulsion with the aid of Span 80 surface-active agent. The mixture was intermixed for 5 min using a magnetic stir plate, Heidolph MR3001 (Heidolph, Germany), at a speed of $1200 \mathrm{rpm}$ to form a stable water in oil emulsion. The emulsion was then further mixed using an ultrasonic processor (Vibra Cell ${ }^{\mathrm{TM}}$ VCX 750, Sonics and Materials Inc., USA) at 300 Watt with the ultrasonic amplitude at $80 \%$ for 1 min to break down the emulsion into smaller droplets.

The water in oil emulsion was then stirred at $20^{\circ} \mathrm{C}$ while adding formaldehyde (ACS Reagent, Sigma Aldrich) to crosslink the microcapsules for $2 \mathrm{~h}$ at $1200 \mathrm{rpm}$. The addition of formaldehyde should be as slow as possible in order to keep the stability of system. Afterwards, precipitation process
B

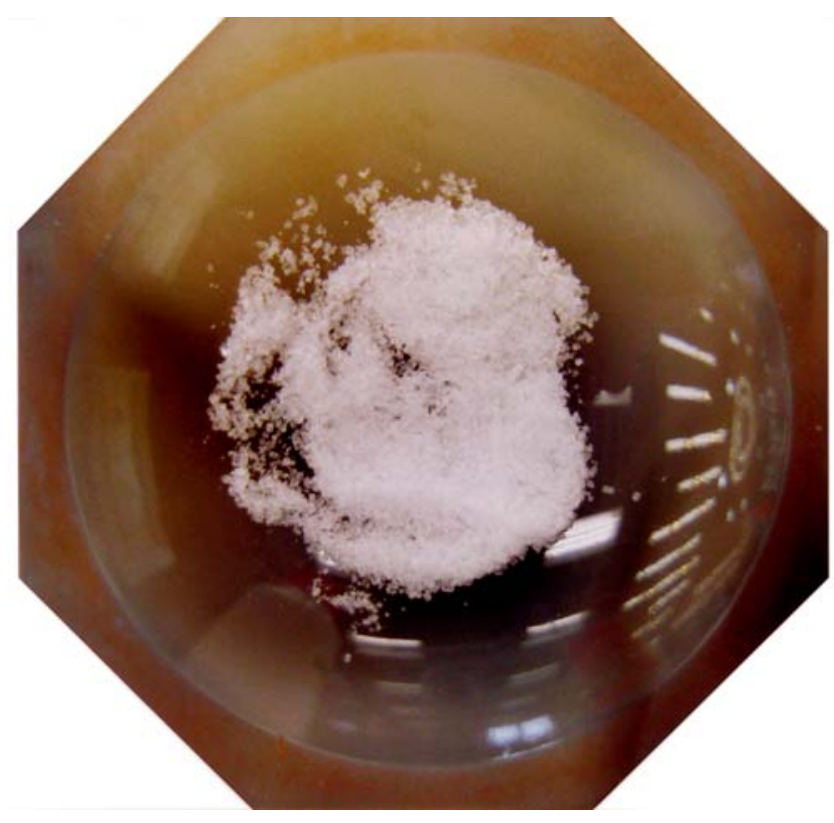

Figure 6. (A) Gelatin powder, (B) vitamin C powder and (C) gelatin/ vitamin $\mathrm{C}$ microcapsules.

was conducted by adding acetone into the solution. The addition of acetone was also aimed at microcapsule separation and dehydration. The precipitates in the form of gelatin microcapsules containing vitamin $\mathrm{C}$ were filtered followed by drying at room temperature as shown in Fig. 6C.

Surface morphology, particle size distribution and toxicity studies of gelatin/vitamin C microcapsules. Optical Microscopy (Nikon HFX-DX, Nikon, Japan) and Scanning Electron Microscopy (SEM) (JEOL JSM 6490, JEOL, Japan) were employed to investigate the surface morphology of microcapsules, degree of agglomeration and particle size distribution. The mean particle size of microcapsules was evaluated using a Zetasizer 3000 HAS (Malvern Instruments, UK). In view of formaldehyde being used as the crosslinking agent in the microencapsulation system, the toxicity studies of gelatin microcapsules was conducted on human liver and breast cell line as well as well as the primary culture obtained from the patient with non-malignant haematological disorder.

Surface morphology and particle size distribution. Fig. 7 shows the optical micrograph of gelatin microcapsules containing vitamin $\mathrm{C}$. The gelatin microcapsules were in spherical form with a certain degree of agglomeration. Fig. 8 shows the SEM image of gelatin microcapsules at the magnification of $x 350$. In agreement with the observation obtained from the optical micrograph, the gelatin microcapsules appeared to be round in shape with relatively smooth surface and the particle size of microcapsules was 


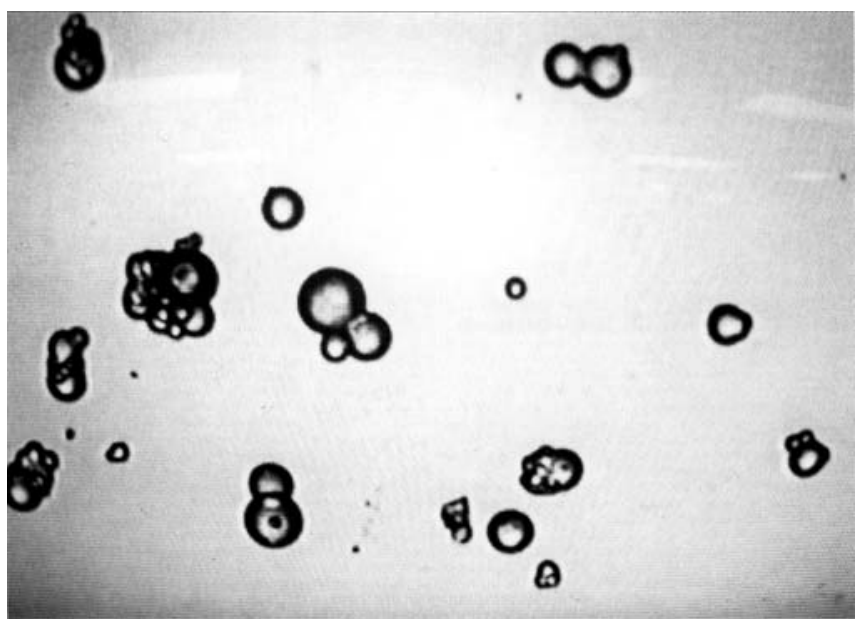

Figure 7. Optical image of gelatin microcapsules containing vitamin C.

ranging from 5.0 to $44.1 \mu \mathrm{m}$. The result of average particle size was $24.6 \mu \mathrm{m}$ as obtained from the particle size analyser. However, a certain degree of agglomeration of microcapsules was noted as shown in Fig. 7. The agglomeration of microcapsules was largely affected by the stirring speed during the cross-linking process. When the stirring speed was too slow, the uncrosslinked microcapsules could not be entirely separated and it would be very difficult to separate the microcapsules once they combined. Hence, high stirring speed during the crosslinking process should be maintained in order to form the separated microcapsules.

Toxicity studies. Formaldehyde is known to be toxic, allergenic and carcinogenic. It is classified as a probable human carcinogen by the US Environmental Protection Agency. Melamine-formaldehyde microcapsules have been studied by a number of researchers over the past decade to encapsulate different essential oils for specific purposes (24,29-32). However, the related cytotoxicity studies of the microcapsules are always missing. Since formaldehyde was used as the crosslinking agent in the gelatin-based microencapsulation system, an in vitro toxicity test of gelatin microcapsules was conducted on the human liver Hep3B and human breast MDAMB-231 cell lines as well as the primary bone marrow culture obtained from patient with nonmalignant haematological disorder. It was observed that these microcapsules exerted no significant toxicological effect on those human cells at a maximum concentration of $100 \mu \mathrm{g} / \mathrm{ml}$ after $48 \mathrm{~h}$ of incubation as shown in Fig. 9A-F, respectively (33-38). The gelatin microcapsules containing vitamin $\mathrm{C}$ were therefore proved to be non-cytotoxic to human cells under in vitro condition.

Application of gelatin/vitamin C microcapsules on textile materials. Cotton $(100 \%)$ fabric samples were cut into many pieces of $20 \times 20 \mathrm{~cm}$. Gelatin microcapsules containing vitamin $\mathrm{C}$ were firstly diluted with deionised water at the liquor ratio of 1:10. The solution was stirred for $10 \mathrm{~min}$ using a magnetic stir plate at a speed of $1200 \mathrm{rpm}$ to obtain a better dispersion of microcapsules. Textile binder (Devan Chemicals) was used to enhance the fixation of gelatin microcapsules

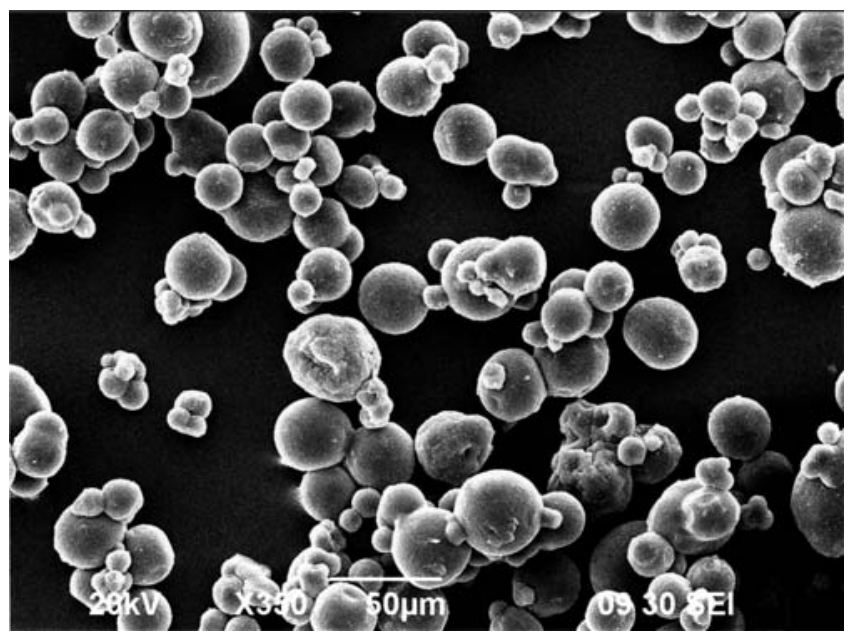

Figure 8. SEM image of gelatin microcapsules containing vitamin C.
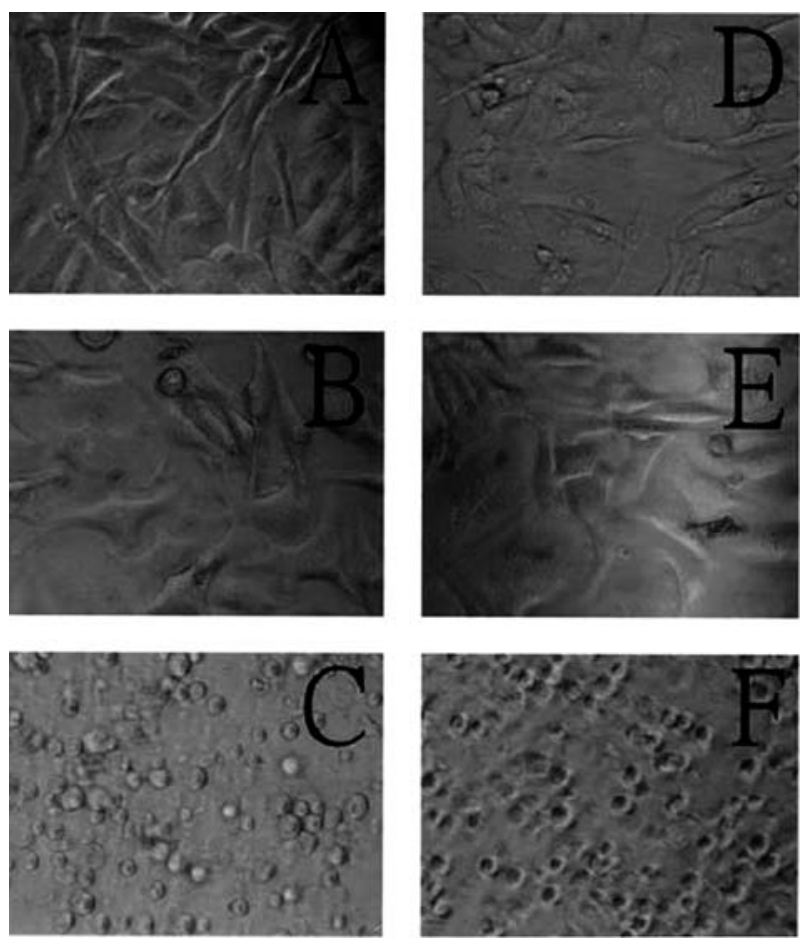

Figure 9. (A) Control liver Hep3B cell line. (B) Control breast MDAMB231 cell line. (C) Control primary culture obtained from patient with nonmalignant haematological disorder. (D) Liver Hep3B cell line treated with gelatin/vitamin C microcapsules. (E) Breast MDAMB-231 cell line treated with gelatin/vitamin C microcapsules. (F) Primary culture obtained from patient with non-malignant haematological disorder treated with gelatin/ vitamin $\mathrm{C}$ microcapsules.

onto the cotton fabric. The fabric samples were then immersed into the microcapsule solution followed by padding using a vertical padder at a constant pressure of $1.5 \mathrm{~kg} / \mathrm{cm}^{2}$ and a speed of $7.5 \mathrm{rpm}$. The wet uptake level of fabric samples was $70 \pm 5 \%$ followed by drying the fabric at room temperature.

Fig. 10A shows the SEM image of cotton fibres exhibiting the fibrous structure. Fig. 10B shows the SEM image of cotton fibres embedded with the gelatin microcapsules. The SEM results confirmed that microcapsules 
A

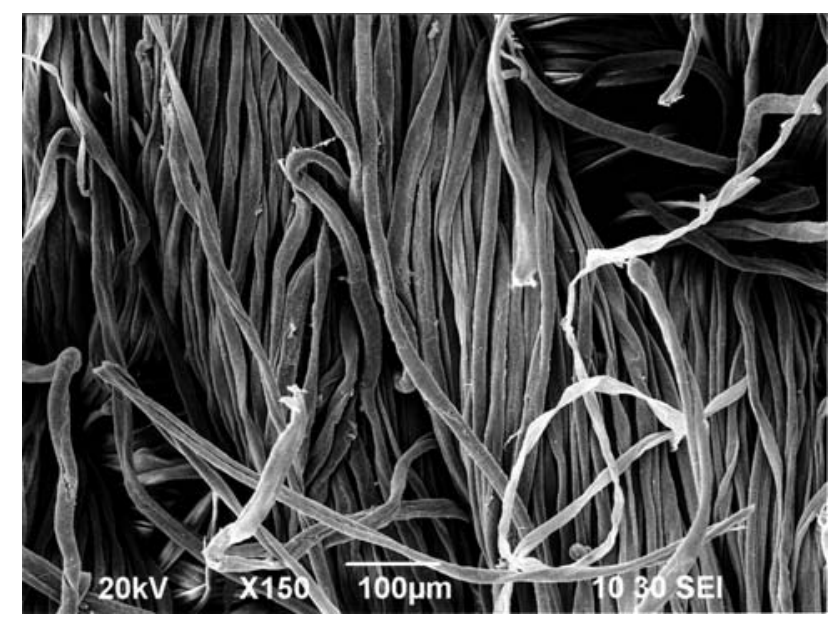

B

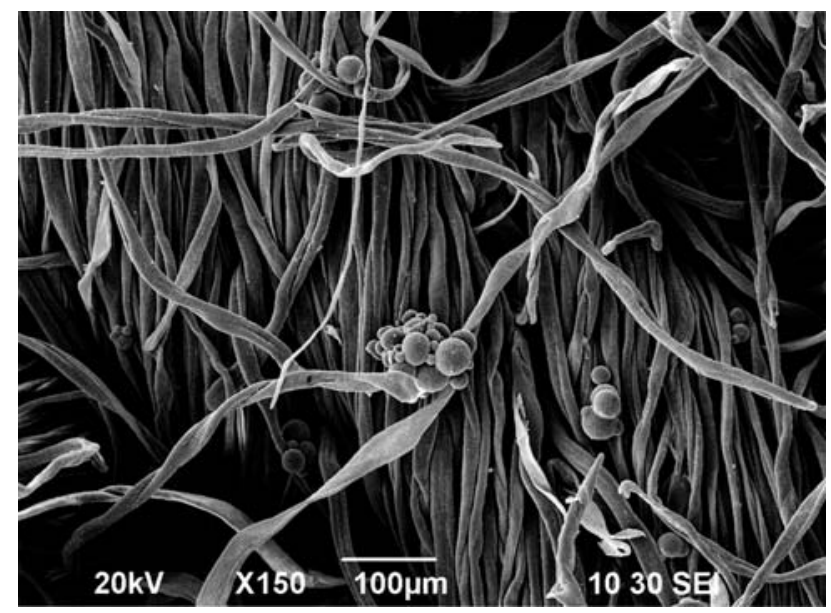

Figure 10. (A) Control cotton fibre and (B) cotton fibre embedded with gelatin/vitamin C microcapsules.

were successfully padded onto the fibre surface without obvious breakage during the padding process. The particle size of the gelatin microcapsules ranged from 6.7 to $36.7 \mu \mathrm{m}$ which was similar to that of the previous SEM evaluation. The use of vitamin $\mathrm{C}$ is known to be beneficial to skin such as promoting the synthesis of collagen and restraining the decomposition of collagen, while it is effective in preventing wrinkles on the skin. The vitamin $\mathrm{C}$ substance is released from the gelatin wall shell in the presence of humidity due to its sensitivity to moisture. A relatively small amount of water is sufficient to achieve the release, such as the water and sweat present on the surface of the skin. Hence, the released vitamin $\mathrm{C}$ may be directly be adsorbed by the skin.

\section{Conclusion}

Cosmetic textiles are increasingly popular and expanding considerably in the textile industry as the market message becomes more widely appreciated. The innovative development of cosmetic textiles can be as wide as imagination. In this study, the gelatin microcapsules containing vitamin $\mathrm{C}$ were prepared using the emulsion hardening technique and successfully grafted into fibrous materials. This provides a practical example for explaining the development of cosmetic textiles with biological benefits. The controlled release mechanism of cosmetic textiles with respect to human skin can be further studied.

The integration of aromatherapy in textile application is a novel and user-friendly idea that enables an alternative basis for essential substance delivery systems. At present, the application of aromatherapy in textiles is commonly concentrated on skin caring benefit and stress management. More innovative ideas can be as wide as imagination such as hair care and treatment, body slimming and even medical applica-tions etc. Microencapsulation technology is an effective technique to achieve satisfactory performance; although it is still relatively new to the textile and apparel industry. The wide range of benefits of aromatherapy and the controlled release of essential oils or active ingredients are expected to be appreciated by consumers.
The application of cosmetic textiles can also be expanded to the bio-medical field. Traditionally, people with adverse skin conditions are treated with either oral or topical drugs, or a combination of both. The traditional problems associated with the use of biological agents on skin are usually related to inappropriate, delayed or missing doses. The development of bio-medical textiles with the microencapsulated agents will definitely offer a more reliable and durable protocols for providing skin-care solutions and treating skin diseases. The events will be focused on encapsulating drugs to provide a controlled release of an active ingredient to solve the skinrelated problems by absorption through the skin. As for the skin-care purposes, the encapsulated components can include i) skin moisturisers, e.g. elastin and keratin, ii) UV blocking agents, e.g. zinc oxide and titanium oxide and iii) growth factors for stimulating hair growth. In the case of treating skin associated disorders, the encapsulated substances can include anti-allergic agents, e.g. anti-histamine, or immunosuppressing agents.

As a whole, it is anticipated that the development of cosmetic textiles will continue to grow and explore completely new possibilities for providing bioactive body care functions to wearer in the near future. It is really a challenging and exciting time for both the textile and medical industries. The textile industry collaborated with medical profession should continue to explore and develop functional textiles that fit the consumer needs and behaviour.

\section{Acknowledgements}

We would like to acknowledge the Central Research Grant provided by The Hong Kong Polytechnic University (Project Code: RGTG and G-U608) for undertaking the research study. This article forms part of the Ph.D. thesis study of S.Y. Cheng.

\section{References}

1. Kan CW and Yuen CWM: Cosmetic textiles. Text Asia 36: 29-35, 2005.

2. Holme I: Innovative technologies for high performance textiles. Color Technol 123: 59-73, 2007. 
3. Cheng SY, Yuen CWM, Kan CW and Cheuk KKL: Imparting cosmetic effects on textiles. Colourage 55: 68-78, 2008.

4. Achwal WB: Textiles with cosmetic substances. Colourage 50: 41-42, 2003.

5. Saez V, Hernandez JR and Peniche C: Microspheres as delivery systems for controlled release of peptides and proteins. Biotechnologia Aplicada 24: 108-116, 2007.

6. Nelson G: Microencapsulation in textile finishing. Rev Prog Color 31: 57-64, 2001.

7. Mei WP: Application of microencapsulation technology in textile coloration of finishing. J China Text Inst 5: 188-191, 1995.

8. Simon B: Microencapsulation: methods and industrial applications. CRC Press, New York, 2006.

9. Anon: Microencapsulation: For enhanced textile performance. Perform Apparel Market 12: 21-39, 2005.

10. Holme I: Versatile technology comes of age. Int Dyer 188: 9-13, 2003.

11. Sudha S, Dev VRG and Neelakandan R: Microencapsulation: An overview. Indian Text J 115: 25-29, 2005.

12. Aggarwal AK, Dayal A and Kumar N: Microencapsulation processes and applications in textile processing. Colourage 45: $15-24,1998$.

13. Erkan G and Sariisik M: Microencapsulation in textiles. Colourage 51: 61-64, 88, 2004.

14. Fisher G: Medical and hygiene textiles-Continuing in good health. Tech Text Int 11: 10-16, 2002.

15. Swerev M: Biofunctional textiles on market within 3-5 years. Med Text Jan: 11, 2001.

16. Kan CW, Yuen CWM and Lai OYA: Aromatherapy in textiles. Text Asia 36: 35-38, 2005

17. Atmane M: Flavor encapsulation and controlled release - a review. Int J Food Sci Technol 41: 1-21, 2006.

18. Holme I: Microencapsulation: the changing face of finishing. Text Mag 31: 7-10, 2004.

19. Holme I: Microencapsulation for novel finishes. Africa and Middle East Text 1: 22-23, 2004.

20. Milmo S: Trends and developments in chemicals for the technical textiles Industry. Tech Text Markets 64: 70-93, 2006.

21. Yamato Y, Yoshida T, Kikuchi M, Okamoto M, Miyoshi K, Fukuda S, Fuse T, Yamauchi T, Ogawa Y, Mutagami S, Shiomura S and Mizukami Y: Microcapsule, treating liquids containing the same, and textile structure having microcapsules adhering thereto. US Patent $005232769,1993$.

22. Bishop JRP, Nelson G and Lamb J: Microencapsulation in yeast cells. J Microencapsul 15: 761-773, 1998.

23. Copete Vidal T, Subirana RP and Capdevila AT: Equipped fibers and textile surface structures. US Patent 0150056 A1, 2005.

24. Hong K and Park S: Melamine resin microcapsules containing fragrant oil: synthesis and characterization. Mater Chem Phys 58: $128-131,1999$.

25. Hong $\mathrm{K}$ and Park S: Preparation of poly(L-lactide) microcapsules for fragrant fibre and their characteristics. Polymer 41: 4567-4572, 2000.

26. Jafari MHS, Parvinzadeh M and Najafi F: Preparation of fragrant microencapsules anal coating on textiles. Nanotechnology and its applications. AIP Conf Proc 929: 224-227, 2007.
27. Li S, Boyter H and Qian L: UV curing for encapsulated aroma finish on cotton. J Text Inst 96: 407-411, 2005.

28. Chang SY: Process of fabric treatment with natural oil essence microcapsule. US Patent 0257324 A1, 2005.

29. Sohn SO, Lee SM, Kim YM, Yeum JH, Choi JH and Ghim HD: Aroma finishing of PET fabrics with PVAc nanoparticles containing lavender oil. Fiber Polym 8: 163-167, 2007.

30. Lee HY, Lee SJ, Cheong IW and Kim JH: Microencapsulation of fragrant oil via in situ polymerization: effects of $\mathrm{pH}$ and melamine-formaldehyde molar ratio. J Microencapsul 19: 559569,2002

31. Hwang JS, Kim JN, Wee YJ, Yun JS, Jang HG, Kim SH and Ryu HW: Preparation and characterization of melamineformaldehyde resin microcapsules containing fragrant oil. Biotechnol Bioprocess Eng 11: 332-336, 2006.

32. Hwang JS, Kim JN, Wee YJ, Jang HG, Kim SH and Ryu HW: Factors affecting the characteristics of melamine resin microcapsules containing fragrant oils. Biotechnol Bioprocess Eng 11: 391-395, 2006.

33. Chan SH, Lam KH, Chui CH, Gambari R, Yuen MCW, Wong RSM, Cheng GYM, Lau FY, Au YK, Chen CH, Lai PBS, Kan CW, Kok SHL, Tang JCO and Chan ASC: The preparation and in vitro antiproliferative activity of phthalimide based ketones on MDAMB-231 and SKHep-1 human carcinoma cell lines. Eur J Med Chem 44: 2736-2740, 2009.

34. Lam KH, Gambari R, Yuen MCW, Kan CW, Chan P, Xu JL, Tang WJ, Chui $\mathrm{CH}$, Cheng GYM, Wong RSM, Lau FY, Tong SCW, Chan AKW, Lai PBS, Kok SHL, Cheng CH, Chan ASC and Tang JCO: The preparation of 2,6-disubstituted pyridinyl phosphosine oxides as novel anticancer agents. Bioorg Med Chem Lett 19: 2266-2269, 2009.

35. Lu GL, Ho CL, Wang Q, Wong WY, Chui CH, Gambari R, Lau FY, Yuen MCW, Tong CSW, Chan AKW, Tang JCO, Ho KP and Cheng GYM. Synthesis and characterization of some metal complexes of 4,5-Diazafluoren-9-one and their biological effects on human carcinoma cells. Aust J Chem 61: 975-980, 2008.

36. Kok SHL, Wong RSM, Gambari R, Cheung F, Lam WS, Lau FY, Cheng GYM, Cheng CH, Lam KH, Chan SH, Tang JCO, Chui $\mathrm{CH}$ and Ho KP: In vitro cytotoxicity of (-)-EGCG octaacetate on MDAMB-231 and SKHep-1 human carcinoma: A pharmaceutical consideration on prodrug design. Int $\mathbf{J}$ Mol Med 22: 841-845, 2008.

37. Kok SHL, Gambari R, Chui CH, Yuen MCW, Lin E, Wong RSM, Lau FY, Cheng GYM, Lam WS, Chan SH, Lam KH, Cheng CH, Lai PBS, Yu MWY, Cheung F, Tang JCO and Chan ASC: Synthesis and anti-cancer activity of benzothiazole containing phthalimide on human carcinoma cell lines. Bioorg Med Chem 16: 3626-3631, 2008.

38. Kok SHL, Gambari R, Chui CH, Lau FY, Cheng GYM, Lai PBS, Lam WS, Chan ASC, Cheng CH, Teo ITN, Yu MWY, Tang JCO, Cheung F, Wong RSM. Paradoxical proliferative potential of iron (II) sulphate on cancer cells after the 3-(4,5-dimethylthiazol-2-yl)-5-(3-carboxymethoxyphenyl)-2-(4-sulfophenyl)2H-tetrazolium (MTS) assay. Int J Mol Med 19: 971-975, 2007. 